\title{
BACTERIOLOGICAL PROFILE OF CELL PHONES OF HEALTHCARE WORKERS AT TERTIARY CARE HOSPITAL.
}

\author{
Dr. Chinjal A. Panchal, Dr. Mitesh N. Kamothi, Dr. Sanjay J. Mehta,
}

1. Post Graduate Student, Department of Microbiology, C.U. Shah Medical College \& Hospital, Surendranagar.

2. Assistant Professor, Department of Microbiology, C.U. Shah Medical College \& Hospital, Surendranagar.

3. Professor \& Head, Department of Microbiology, C.U. Shah Medical College \& Hospital, Surendranagar.

\section{CORRESPONDING AUTHOR,}

Dr. Chinjal Arvindbhai Panchal,

C. U. Shah Medical College and Hospital,

Surendranagar - 363001,

E-mail: drchinjal@gmail.com,

Ph: 09898576504.

ABSTRACT: BACKGROUND AND OBJECTIVES: Cell phones are increasingly used by healthcare workers. They can harbour potential bacterial pathogens and can become an exogenous source of nosocomial infections. Aim of this study was to identify microbial colonisation and their antimicrobial sensitivity; from cell phones used by healthcare workers.

MATERIALS AND METHODS: A total of 100 samples were collected from cell phones of clinical and para-clinical staff and non-medical personnel. Specimens were collected and subjected to culture and sensitivity as per the standard guidelines.

RESULTS: Bacterial species were isolated from 65 (65\%) out of total 100 samples. Coagulase Negative Staphylococcus (42) was the most commonly isolated organism. Isolates included Methicillin resistant Staphylococcus aureus (10), Methicillin sensitive Staphylococcus aureus (3), Bacillusspp(15), Pseudomonas spp(4),Moraxella spp(2), E. coli (1), Klebsiella spp (1) and Acinetobacterspp (1).

CONCLUSION: The carriage rate of bacterial isolates was higher in the clinical staff (92\%) in comparison to para-clinical staff (56\%) and non-medical personnel $(20 \%)$. Cell phones may serve as potential source of nosocomial infections.

KEYWORDS: Bacterial pathogens, Multidrug resistance, Healthcare workers, Nosocomial infections

INTRODUCTION: The global system for mobile telecommunication (GSM) was established in 1982 in Europe with a view of providing and improving communication network. Cell phones have become one of the most indispensable accessories of professional and social life.1Today,India has 500million Cell phone users. Cell phones increase the speed of communication and contact within health care institutions, making health care delivery more efficient; hence, Cell phones are increasingly used by healthcare workers. ${ }^{2}$ Cell phones are frequently used in hospital wards, casualty, laboratories, ICUs and operation theatres. ${ }^{3}$ However, Cell phones which are seldom cleaned and often touched during or after the examination of patients without hand washing, can harbour various potential pathogens and become an exogenous source of nosocomial infections for hospitalized patients. ${ }^{4}$ Nosocomial infections are important problem in all hospitals. These infections are increasing day-by-day and can affect rate of mortality and morbidity. In this study, Cell phones of non-medical personnel were also included as it represents an environment of community. Therefore bacterial isolates from Cell

Journal of Evolution of Medical and Dental Sciences/Volume1/ Issue3/July-Sept 2012 Page 198 
phones of HCWs may vary in numbers and antibiotic sensitivity, to that found on Cell phones of non-medical personnel. ${ }^{5}$

MATERIALS AND METHODS: The study was conducted at the Department of Microbiology, C. U. Shah Medical College and Hospital, Surendranagar, Gujarat fromAugust 2011 to March 2012.

1) COLLECTION OF SAMPLES: Total of 100 Cell phones of healthcare workers of clinical and para-clinical staff and non-medical personnel were randomly selected for the study.Sterile swabs moistened with peptone water were used to collect specimens from the front, sides and back of the Cell phones.

2) PROCESSING OF SAMPLES: Swabs taken from Cell phones were inoculated on nutrient agar, blood agar and MacConkey agar. The culture plates were incubated at $37^{\circ} \mathrm{C}$ for 24 hours. Isolation and identification of bacterial species were done as per standard guidelines. Antimicrobial susceptibility testing, including detection of Methicillin Resistant Staphylococcus aureus (MRSA) and Extended Spectrum ß-Lactamase (ESBL) was performed for potential pathogenic isolates by disc diffusion method as per the Clinical and Laboratory Standards Institute (CLSI) guidelines. ${ }^{6}$

RESULTS: Out of 100 Cell phones, bacterial growth was obtained in 65 (65\%) Cell phones; which includes 46 from clinicalstaff, 14 from para-clinical staff and 5 from non-medical personnel; as shown inChart-1. The carriage rate was $92 \%$ in clinical staff, $56 \%$ in para-clinical staff and $20 \%$ from non-medical personnel.

Out of 65 Cell phones which yielded bacterial growth, 51 (78.46\%) had single bacterial isolate and $14(21.54 \%)$ had two bacterial isolates making total of 79 isolates.

Out of total 79 isolates, 42 were Coagulase Negative Staphylococci (CONS), 15 were Bacillusspp, 13 were S. aureus and 4 were Pseudomonas spp. Various organisms isolated from clinical, para-clinical and non-medical personnel are shown in Table-1.

Out of $13 \mathrm{~S}$. aureus, 10 and 42 CONS,21were Methicillin resistant, as shown inChart-2.AllE. coli (1), Klebsiella $\operatorname{spp}(1)$, Pseudomonasspp(4) and Acinetobacterspp (1)isolates were multidrug resistant as shown in Table $\mathbf{- 2}$.

\section{Chart - 1Growth obtained from Cell phones.}

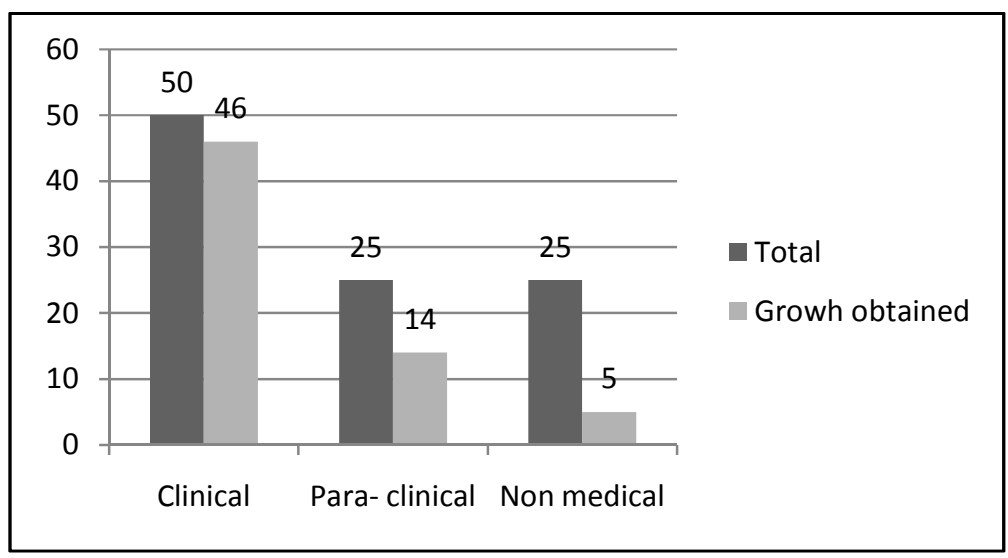

Journal of Evolution of Medical and Dental Sciences/Volume1/ Issue3/July-Sept 2012 Page 199 
Table - 1 Distribution of bacterial isolates

\begin{tabular}{|c|c|c|c|c|}
\hline Organism & Clinical & Para - clinical & Non medical & Total \\
\hline CONS & $\begin{array}{c}29 \\
(69.04 \%)\end{array}$ & $\begin{array}{c}11 \\
(26.19 \%)\end{array}$ & $\begin{array}{c}2 \\
(4.76 \%)\end{array}$ & 42 \\
\hline Bacillus sp. & $\begin{array}{c}10 \\
(66.67 \%)\end{array}$ & $\begin{array}{c}1 \\
(6.66 \%)\end{array}$ & $\begin{array}{c}4 \\
(26.67 \%) \\
\end{array}$ & 15 \\
\hline S. aureus & $\begin{array}{c}10 \\
(76.92 \%)\end{array}$ & $\begin{array}{c}3 \\
(23.08 \%)\end{array}$ & 0 & 13 \\
\hline $\begin{array}{l}\text { Pseudomonas } \\
\text { sp. }\end{array}$ & $\begin{array}{c}4 \\
(100 \%)\end{array}$ & 0 & 0 & 4 \\
\hline Moraxella sp. & $\begin{array}{c}2 \\
(100 \%)\end{array}$ & 0 & 0 & 2 \\
\hline $\begin{array}{l}\text { Acinetobacter } \\
\text { sp. }\end{array}$ & $\begin{array}{c}1 \\
(100 \%)\end{array}$ & 0 & 0 & 1 \\
\hline Klebsiella sp. & $\begin{array}{c}1 \\
(100 \%)\end{array}$ & 0 & 0 & 1 \\
\hline E. coli & $\begin{array}{c}1 \\
(100 \%)\end{array}$ & 0 & 0 & 1 \\
\hline Total & $\begin{array}{c}58 \\
(73.42 \%)\end{array}$ & $\begin{array}{c}15 \\
(18.99 \%)\end{array}$ & $\begin{array}{c}6 \\
(7.59 \%)\end{array}$ & 79 \\
\hline
\end{tabular}

Chart - 2 Methicillin resistance among Staphylococcus species

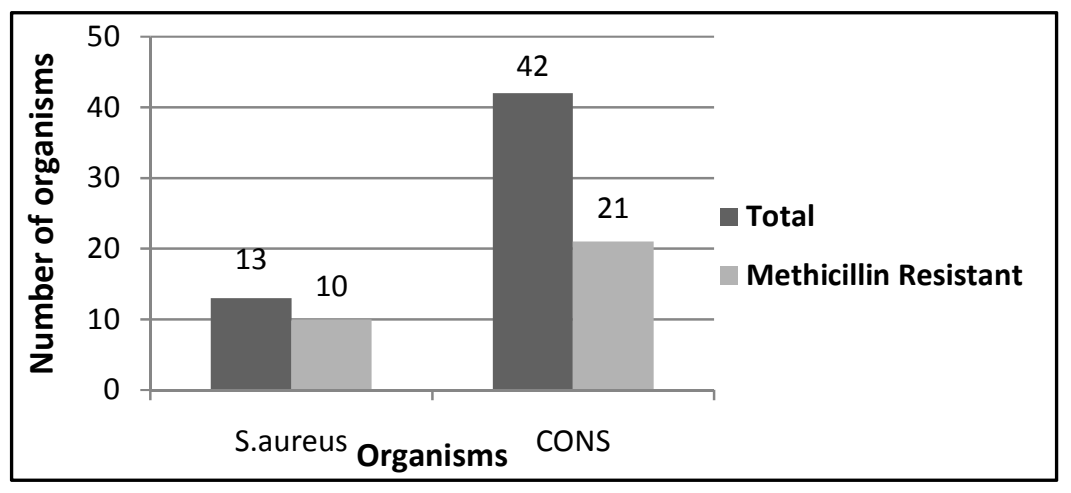

Table - 2Percentage incidence of antibiotic sensitivity of bacterial isolates

\begin{tabular}{|c|c|c|c|c|c|c|c|c|c|}
\hline Organism & Total & AS & BA & CH & RC & TE & GM & $\mathbf{C X}$ & CF \\
\hline CONS & 42 & $\begin{array}{c}38 \\
(90.48 \%) \\
\end{array}$ & $\begin{array}{c}23 \\
(54.76 \%) \\
\end{array}$ & $\begin{array}{c}33 \\
(78.57 \%) \\
\end{array}$ & $\begin{array}{c}28 \\
(66.67 \%) \\
\end{array}$ & $\begin{array}{c}30 \\
(71.42 \%) \\
\end{array}$ & $\begin{array}{c}32 \\
(76.19 \%) \\
\end{array}$ & $\begin{array}{c}21 \\
(50 \%) \\
\end{array}$ & $\begin{array}{c}16 \\
(38.09 \%) \\
\end{array}$ \\
\hline S. aureus & 13 & $\begin{array}{c}11 \\
(84.62 \%)\end{array}$ & $\begin{array}{c}5 \\
(38.46 \%)\end{array}$ & $\begin{array}{c}5 \\
(38.46 \%)\end{array}$ & $\begin{array}{c}7 \\
(53.85 \%)\end{array}$ & $\begin{array}{c}7 \\
(53.85 \%)\end{array}$ & $\begin{array}{c}9 \\
(69.23 \%)\end{array}$ & $\begin{array}{c}3 \\
(23.08 \%)\end{array}$ & $\begin{array}{c}3 \\
(23.08 \%)\end{array}$ \\
\hline $\begin{array}{l}\text { Pseudomonas } \\
\text { spp }\end{array}$ & 4 & $\begin{array}{c}2 \\
(50 \%)\end{array}$ & $\begin{array}{c}1 \\
(25 \%)\end{array}$ & $\begin{array}{c}1 \\
(25 \%)\end{array}$ & $\begin{array}{c}1 \\
(25 \%)\end{array}$ & - & $\begin{array}{c}3 \\
(75 \%)\end{array}$ & $\begin{array}{c}1 \\
(25 \%)\end{array}$ & - \\
\hline $\begin{array}{l}\text { Moraxella } \\
\text { spp }\end{array}$ & 2 & $\begin{array}{c}1 \\
(50 \%)\end{array}$ & $\begin{array}{c}1 \\
(50 \%)\end{array}$ & $\begin{array}{c}1 \\
(50 \%)\end{array}$ & $\begin{array}{c}1 \\
(50 \%)\end{array}$ & - & $\begin{array}{c}1 \\
(50 \%)\end{array}$ & - & - \\
\hline Klebsiella spp & 1 & $\begin{array}{c}1 \\
(100 \%)\end{array}$ & - & $\begin{array}{c}1 \\
(100 \%)\end{array}$ & $\begin{array}{c}1 \\
(100 \%)\end{array}$ & - & $\begin{array}{c}1 \\
(100 \%)\end{array}$ & - & - \\
\hline E. coli & 1 & $\begin{array}{c}1 \\
(100 \%)\end{array}$ & $\begin{array}{c}1 \\
(100 \%)\end{array}$ & $\begin{array}{c}1 \\
(100 \%)\end{array}$ & $\begin{array}{c}1 \\
(100 \%)\end{array}$ & - & $\begin{array}{c}1 \\
(100 \%)\end{array}$ & - & - \\
\hline $\begin{array}{l}\text { Acinetobacter } \\
\text { spp }\end{array}$ & 1 & $\begin{array}{c}1 \\
(100 \%) \\
\end{array}$ & - & - & $\begin{array}{c}1 \\
(100 \%) \\
\end{array}$ & $\begin{array}{c}1 \\
(100 \%) \\
\end{array}$ & $\begin{array}{c}1 \\
(100 \%) \\
\end{array}$ & - & - \\
\hline
\end{tabular}


AS - Ampicillin/ Sulbactum,CH - Chloramphenicol,TE - Tetracycline,CX -Cefoxitn,

BA - Cotrimoxazole,RC - Ciprofloxacin,GM - Gentamycin,CF - Ceftiaxone

DISCUSSION: The study shows that bacterialpathogensarepresent oncellphones. The carriage rate of bacterial isolates was $65 \%$, which was higher in clinical (92\%) staff than in paraclinical (56\%) staff and non- medical personnels (20\%). Similar pattern was observed by Arora U. et al 2. Table-3 shows carriage ratesof bacterial isolates oncellphones reported by various authors.Bacterial isolates from cellphones of HCWs were varying in their numbers and antibioticsensitivity, to that found on cellphones of non-medical personnels.

Coagulase NegativeStaphylococci(CONS)were found to be the most common colonizing organisms in the present study. Similar pattern have been reported by different authors as shown inTable-4.

Many bacterial pathogens isolated from cellphones of healthcare workers were also multidrug resistant. 21(50\%) Coagulase NegativeStaphylococciand 10 (76.92\%)S. aureuswere methicillin resistant.All E. coli, Klebsiella spp, PseudomonassppandAcinetobactersppwerealsomultidrug resistant.

Table -3 Comparison of carriage rate with other studies

\begin{tabular}{|l|c|c|c|c|}
\hline \multicolumn{1}{|c|}{ Study } & $\begin{array}{c}\text { Organisms } \\
\text { isolated }\end{array}$ & Clinical & $\begin{array}{c}\text { Para - } \\
\text { clinical }\end{array}$ & $\begin{array}{c}\text { Non- } \\
\text { medical }\end{array}$ \\
\hline Kabir O. et al - 2009(1) & $62.00 \%$ & $15.30 \%$ & - & $66.45 \%$ \\
\hline Arora U. et al - 2009(2) & $40.62 \%$ & $44.78 \%$ & $37.77 \%$ & - \\
\hline Jayachandra T. et al - 2011(3) & $84.30 \%$ & - & - & - \\
\hline Jayalakshmi J. et al - 2008(7) & $91.67 \%$ & $90.40 \%$ & $93.30 \%$ & - \\
\hline Bhat S.- 2011(8) & $98.50 \%$ & - & - & - \\
\hline Datta P. - 2009 (9) & $59.60 \%$ & - & - & $10.00 \%$ \\
\hline Present study & $\mathbf{6 5 . 0 0 \%}$ & $\mathbf{9 2 . 0 0 \%}$ & $\mathbf{5 6 . 0 0 \%}$ & $\mathbf{2 0 . 0 0 \%}$ \\
\hline
\end{tabular}

Table - 4 Comparison of CONS carriage rate with other studies

\begin{tabular}{|l|c|}
\hline \multicolumn{1}{|c|}{ Study } & CONS \\
\hline Kabir O. et al - 2009(1) & $42.70 \%$ \\
\hline Arora U. et al - 2009 (2) & $27.55 \%$ \\
\hline Jayalakshmi J. et al-2008 (7) & $47.16 \%$ \\
\hline Bhat S. et al-2011 $(8)$ & $21.78 \%$ \\
\hline Fatma U. et al - 2009 (10) & $58.96 \%$ \\
\hline Present study & $\mathbf{5 3 . 1 6 \%}$ \\
\hline
\end{tabular}

CONCLUSION: The overall carriage rate of Cell phones was found to be $65 \%$ in the present study. It was higher in the clinical staff $(92 \%)$ as compared to para-clinical staff $(50 \%)$ and nonmedical personnel 20 (20\%). Cell phones harboured potential pathogens, which included multidrug resistant strains.

Restriction of Cell phones seems impractical, prevention of nosocomial pathogens is important, use of antibacterial agents to clean Cell phones and strict adherence to infection control measures like hand washing and good hygienic practice can reduce the risk of spread of nosocomial pathogens. 3,9 
ACKNOWLEDGMENT: We are sincerely thankful to the Dean, Dr. H. H. Agravat and Superintendent Dr. K.G. Patel of C.U. Shah Medical College\& Hospital for their permission to carry out this study.

\section{REFERENCES:}

1) Kabir O, Audu D, Olabisi O, Akitoye 0 . The potential roleof mobile phones in spread of bacterial infections.JinfectDevCtries2009; 3(8):628-32.

2) Arora U, Pushpa D, Chadha A, Malhotra S. Cell phones amodernstayhouse for bacterial pathogens. JK Science2009;11(3):127-9.

3) Jayachandra T, LakshmiprasannaT, Venkateswar A. Astudy on isolation and identificationofbacteria causing nosocomial infections on mobile phones of health care workers. CalicutMedical Journal 2011; 9(1):1-6.

4) SrikanthP, Rajaram E,Sudharsanam S, Lakshmanan A, UmamaheswariS, Kalyani J. The mobile phone in a tropical setting - emerging threat for infection control. Sri Ramachandra Journal of Medicine 2009;2(2):18-20

5) Chawla K, Mukhopadhayay C, Gurung B, Bhate P, Bairy I. Bacterial 'Cell' Phones: Do cell phones carry potential pathogens?Online J Health AlliedScs.2009; 8(1):8-12.

6) Clinical and Laboratory Standards Institute. Performance standard for antimicrobial susceptibility testing;20 thinformational supplementM100-S20,Vol 30 No 1 . Clinical and Laboratory Standards Institute, Wayne,PA, USA; 2010.

7) Jayalakshmi J, Appalaraju B, Usha. Cell phonesas reservoirs of nosocomial pathogens. J AssocPhy India 2008;56:388-9.

8) Bhat $S$, Hegde $S$, Salian S. Potential of mobile phones to serve as a reservoir in spread of nosocomial pathogens. Online JHealth AlliedScs. 2011;10(2):14-20.

9) Datta P, Rani H,ChanderJ, Gupta V. Bacterial Contamination of Mobile Phones of HealthCare Workers. Ind J Med Microbiol 2009; 27(3):279-81.

10) Ulger F, Esen S, Dilek A, Yanik K, Gunaydin M, Leblebicioglu H. Are we aware how contaminated our mobile phones with nosocomial pathogens?Annals of Microbiology and Antimicrobials2009,8:7. 